\title{
Integrated Filtering Planar Dipole Antenna Using Edge Coupled Feed
}

\author{
Amira El-Tokhy Ali, Yi Wang \\ Department of Engineering Science, University of Greenwich \\ Chatham Maritime, Kent, ME4 4TB, UK. \\ a.e.a.eltokhy@gre.ac.uk, yi.wang@gre.ac.uk
}

\begin{abstract}
The paper presents a novel uniplanar integrated filtering dipole with a second-order filtering response of enhanced frequency selectivity. The first stage of the filter is a $U$ shaped half-wavelength resonator, whereas the second one is the planar dipole antenna. The bandwidth of the dipole is controlled by the edge coupling between the two stages. The U-shape resonator also acts as a balun. A truncated microstrip ground plane is used as the reflector to achieve a directional radiation pattern. The measured results show a bandwidth of $21 \%$ between 4.2 $\mathrm{GHz}$ and $5.2 \mathrm{GHz}$ with a sharper roll-off than a conventional planar dipole, and an end-fire radiation pattern.
\end{abstract}

Keywords - Integrated filtering antenna, planar dipole, edge coupling.

\section{INTRODUCTION}

Antenna and microwave filters are two essential components in many RF front ends. Integrating the adjacent filter and antenna into one platform has the immediate benefits of reduced component counts and device footprint. Apart from that, the bandwidth and selectivity of the antenna can be controlled or significantly enhanced through a seamless integration approach, where the resonant antenna element is implemented as one resonant pole of a coupled-resonator filter and electromagnetically coupled with the other non-radiating resonators [1]-[9]. The coupling can be used to control the antenna bandwidth whereas the embedded filtering function increases its frequency selectivity. Another benefit of this integration approach is the complete elimination of the $50 \Omega$ interface between the traditionally cascaded filters and antennas, which often causes mismatch and bandwidth degradation [3]. Over the last few years, various antenna-filter integration schemes have been demonstrated, mainly concerning patch antennas [2]-[5], monopoles [1], [6] and slot antennas [7].

In this paper, an integrated filter-dipole is proposed by electromagnetically coupling a resonator with a halfwavelength dipole. A bandwidth of $21 \%$ and enhanced frequency selectivity has been achieved. WLAN frequency band had been targeted. A unique feature of the design is that the resonator also served as a balun to the uniplanar microstrip-line dipole. Traditionally the microstrip dipole is fed either using a uniplanar microstrip-to-coplanar-strip transition [10] or a multi-layer structure with the dipole arms located on the opposite sides of the substrate [11]. Here, an edge coupled uniplanar feeding structure is explored. A truncated microstrip ground is implemented to realize an endfire directional radiation pattern.

\section{DESGIN}

This paper presents an integrated design of a second-order uniplanar filter-dipole using an edge coupling approach. It consists of a U-shape half-wavelength resonator and a planar half-wavelength dipole antenna as a second resonator as shown in Fig. 1. Rogers RO4003C substrate was used with a thickness of $1.524 \mathrm{~mm}$, a dielectric constant of 3.55 and a loss tangent of 0.0027. As shown in Fig. 1, the top surface of the substrate contains the feeding line, the U-shape resonator, and the continuous-line dipole. The bottom surface contains the truncated microstrip ground which also acts as the reflector. An interdigital structure is used between the U-shaped resonator and the feed line in order to achieve strong capacitive coupling, which is required for an enhanced bandwidth. The U-shape resonator is edge-coupled to the halfwavelength dipole at its central point, where the current peaks, through both capacitive and inductive coupling. The width of the dipole $\mathrm{Ld}$ is increased to improve impedance matching. The partial ground is $8.92 \mathrm{~mm} \times 45 \mathrm{~mm}$ in size. It is distanced from the dipole to minimize the adverse effect on directivity. The size of the partial ground has significant impact on both impedance matching and the radiation pattern. This makes the U-shaped resonator only has a partial ground. The total size of the filter-antenna is $30 \mathrm{~mm} \times 60 \mathrm{~mm}$. CST Microwave Studio has been used for the simulations and layout optimisation.
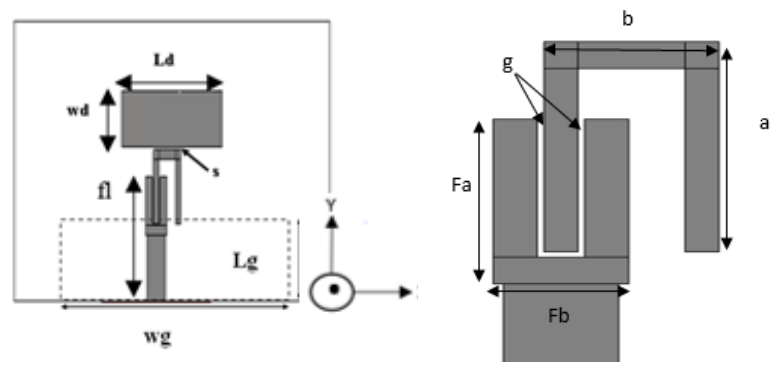

Fig. 1. Integrated filtering dipole antenna using an edge coupled feed. $\mathrm{wd}=6$ $\mathrm{mm}, \mathrm{Ld}=19 \mathrm{~mm}, \mathrm{Lg}=8.92 \mathrm{~mm}, \mathrm{gw}=45 \mathrm{~mm}, \mathrm{fl}=13.3 \mathrm{~mm}, \mathrm{a}=7.92 \mathrm{~mm}, \mathrm{~b}$ $=5.2 \mathrm{~mm}, \mathrm{~g}=0.2 \mathrm{~mm}, \mathrm{Fa}=6.2 \mathrm{~mm}, \mathrm{Fb}=4 \mathrm{~mm}, \mathrm{~s}=0.3 \mathrm{~mm}$. 
A parametric analysis has been performed to study the effects of the dipole size and coupling gaps on the impedance matching. From Fig. 2(a) and (b), it can be seen that a dipole width of $9 \mathrm{~mm}$ and a length of $19 \mathrm{~mm}$ gave an optimal matching condition. Fig. 2(c) and (d) show that the feeding gap $g$ (controlling the external coupling) and the separation $s$ between the dipole and the resonator (controlling the mutual coupling) have a significant influence on the impedance bandwidth.

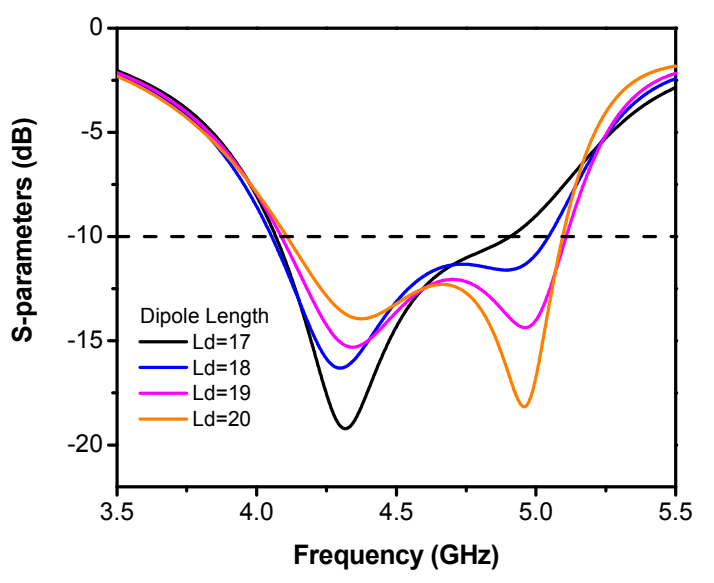

(a)

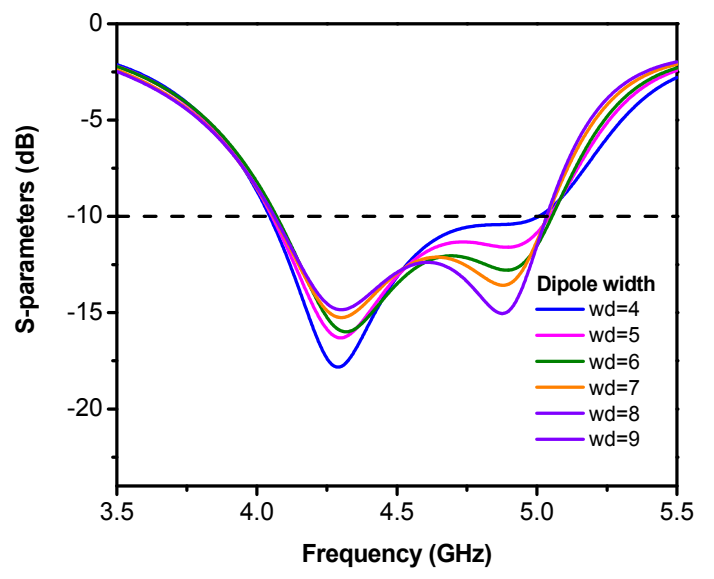

(b)

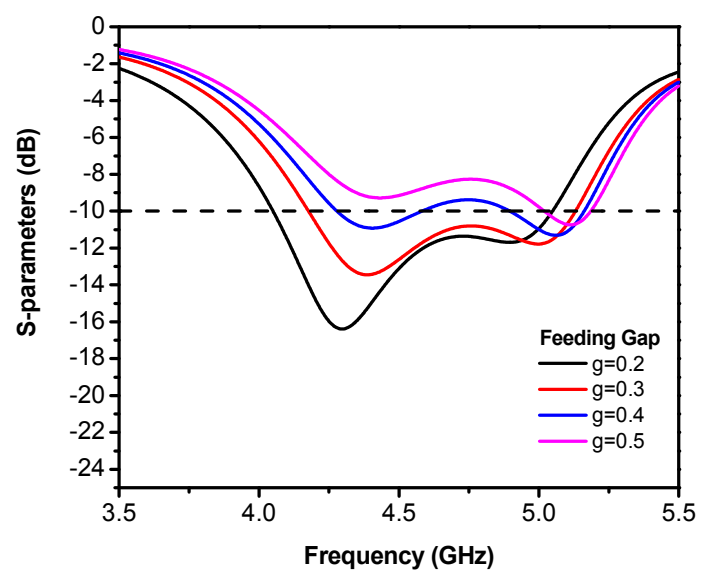

(c)

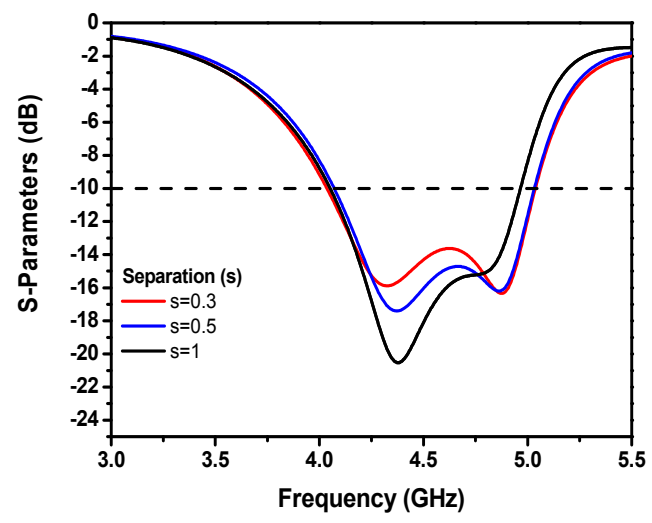

(d)

Fig. 2. The parametric sweep of (a) the planar dipole length, (b) the planar dipole width, (c) the gap between the feed and the resonator, and (d) the separation between the dipole and the resonator.

\section{SimULATION RESUlTS}

Fig. 3, shows the simulated reflection coefficient $\mathrm{S}_{11}$ indicating a bandwidth of $1 \mathrm{GHz}$ between $4.04 \mathrm{GHz}$ and 5.04 $\mathrm{GHz}$ and two reflection dips as in a second-order filter. From the simulated surface current distribution which is shown Fig. 4 , it can be seen that the first resonance dip was mainly attributed to the dipole antenna. The second resonant dip was mainly due to the U-shape resonator. Occupying the same substrate area as the filter-dipole, a conventional microstrip dipole with its two arms printed on the opposite sides of the substrate and without the resonator (as shown by the inset of Fig. 3) was also designed for comparison. As shown in Fig. 3, its bandwidth is $700 \mathrm{MHz}$. The filtering dipole gave a $43 \%$ increase in bandwidth. Fig. 3 also shows that the filtering dipole has a sharper roll-off at the band edges.

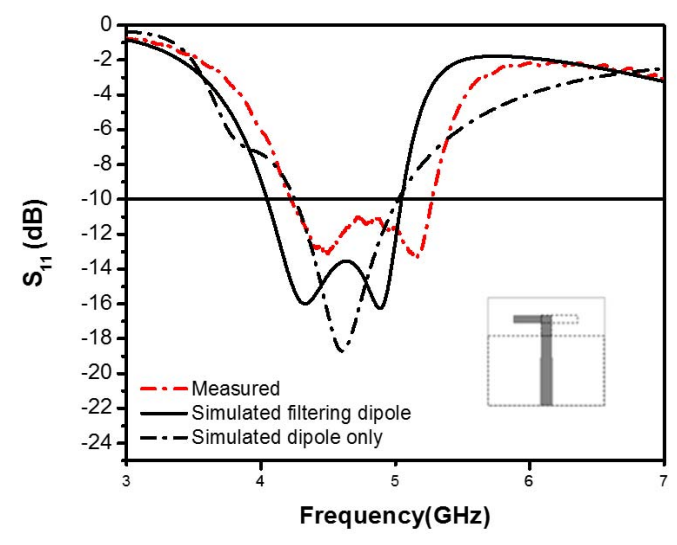

Fig. 3. Simulated and measured $S_{11}$ of the filtering-dipole in comparison with that of a traditional microstrip dipole, the layout of which is shown as the inset. 


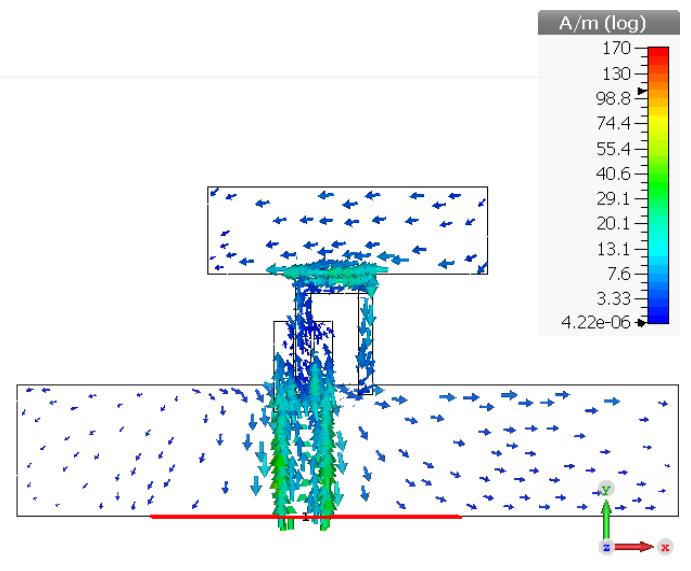

(a)

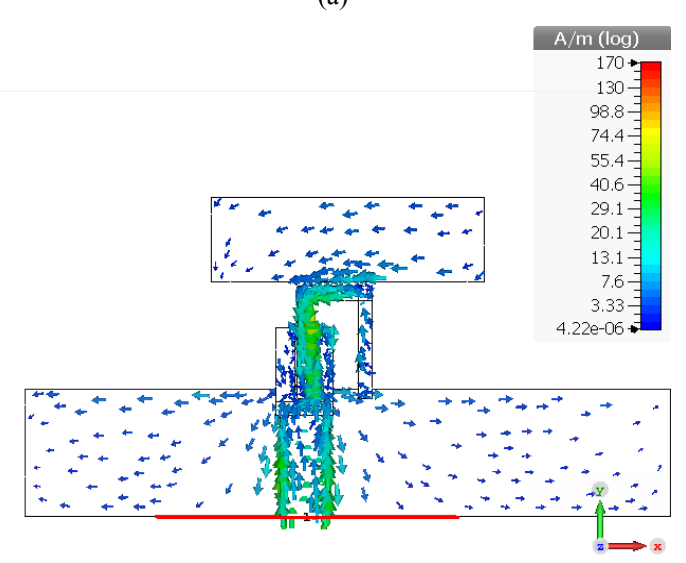

(b)

Fig. 4. The simulated current distribution of the filtering-dipole (a) at 4.3 $\mathrm{GHz}$, and (b) $4.9 \mathrm{GHz}$.

\section{FABRICATIONS AND MEASURMENTS}

The filtering dipole is fabricated using the milling method with an LPKF ProtoMat S63 circuit board plotter. The fabricated antenna is shown in Fig. 5. The measured $S_{11}$ is shown in Fig. 3. There is a shift in the frequency band of 160 $\mathrm{MHz}$ to between $4.2 \mathrm{GHz}$ and $5.2 \mathrm{GHz}$. This is mainly due to the over-milling of the circuit. The measured E-plane (xyplane as indicated in Fig. 1 and 5) radiation patterns at 4.2, 4.7 and $5.2 \mathrm{GHz}$ are shown in Fig. 6, for both co-polarization and cross-polarization. The cross polarization discrimination levels are $22 \mathrm{~dB}, 25 \mathrm{~dB}$ and $32 \mathrm{~dB}$ respectively. It is observed that the main beams were tilted by $25^{\circ}$ to $30^{\circ}$ off the feed line direction. The tilting is attributed to the asymmetric feed to the dipole. The simulated radiation patterns at the simulated band edges $(4.04 \mathrm{GHz}$ and $5.04 \mathrm{GHz})$ and at the centre $(4.54 \mathrm{GHz})$ are also shown for comparison. Good agreement between simulations and measurements has been achieved. The radiation patterns are largely maintained across the band.

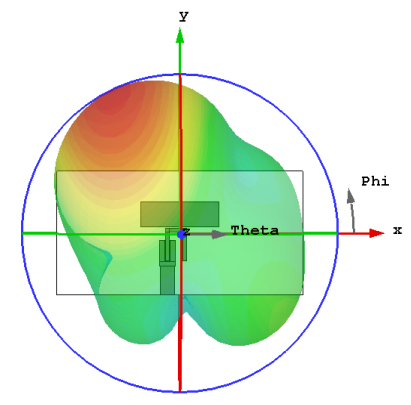

(a)

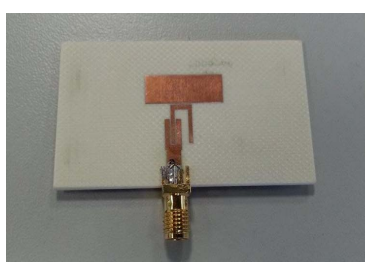

(b)
Fig. 5. Top view of (a) the $3 \mathrm{D}$ radiation pattern at the centre frequency with the allocated coordinates and (b) the fabricated filtering dipole.

The use of the truncated ground helped the filter-dipole to achieve a gain of 4.0-5.0 $\mathrm{dBi}$ across the band. Fig. 7 shows the measured and simulated gains as a function of frequency. Reasonable agreement has been achieved except for the frequency shift. It should be noted that the gains are taken on the E-plane at a fixed angle of $30^{\circ}$, which is close to the main beam direction. The measurement range is limited by the standard gain horn $(3.9$ - $5.9 \mathrm{GHz})$ available in the anechoic chamber. The gain curve shows the rapid roll-off at the band edges, which is a signature of the filtering characteristics of the antenna.

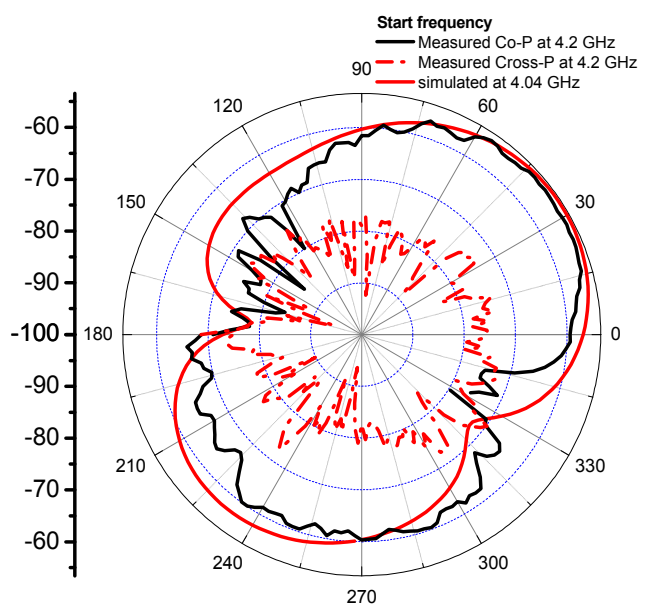

(a) 


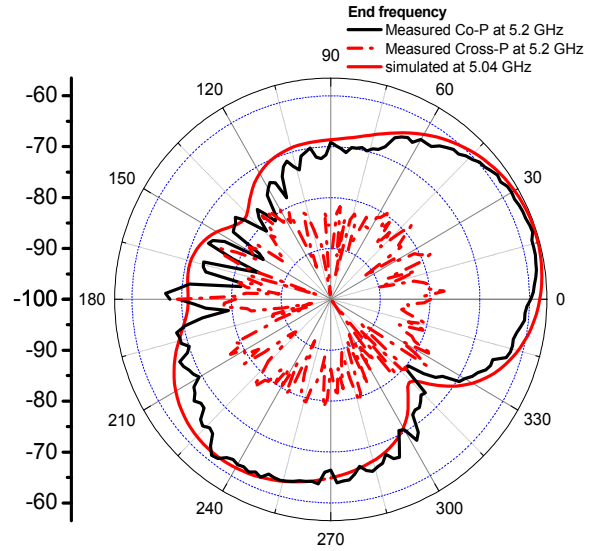

(b)

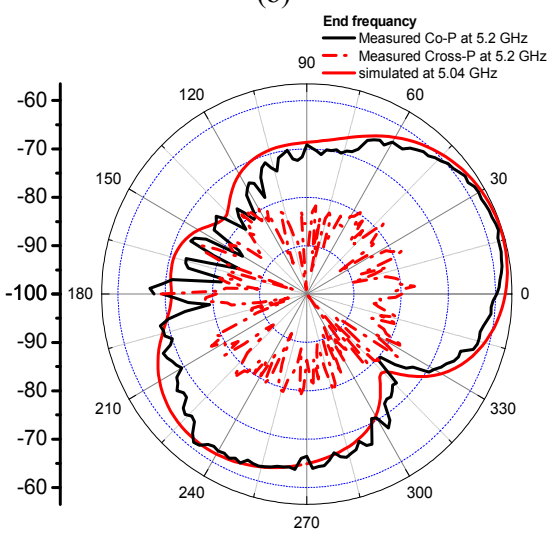

(c)

Fig. 6. Simulated and measured radiation patterns at (a) the start, (b) the center, and (c) the end frequency of the band.

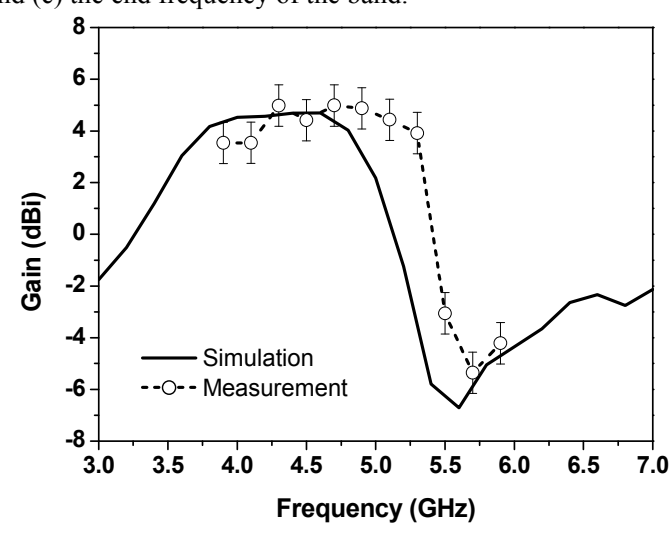

Fig. 7. Measured and simulated gains at $30^{\circ}$ direction of the filtering dipole as a function of frequency.

\section{CONCLUSION}

In this paper an integrated uniplanar filtering dipole antenna has been presented with a $21 \%$ bandwidth and a second-order filtering characteristics. The continuous-line half-wavelength dipole is treated as the second resonator in the filter. The first resonator acts as the balun and edge-coupled to the dipole. This coupling scheme can be favourably used to control the antenna bandwidth and frequency selectivity. The titled main beam and the low front-to-back ratio are the main limitations of the current design. The partial ground of the Ushaped resonator also causes unwanted radiation from the resonator itself. For the future work, the beam may be corrected using a symmetric feeding structure and the directivity can be further improved by using directors as in a Yagi-Uda antenna.

\section{REFERENCES}

[1] W. Wu, Y. Yin, S. Zuo, Z. Zhang, and J. Xie, "A New Compact Filter Antenna for Modern Wireless Communication Systems," IEEE Antennas Wireless Propag. Lett., vol. 10, pp. 1131-1134, 2011.

[2] C.K. Lin, and S.J. Chung, "A Compact Edge-Fed Filtering Microstrip Antenna with 0.2 dB Equal-Ripple Response," in Proc. the 39th Eur. Microw. Conf., pp. 378-380, 2009.

[3] C. Mao, S. Gao, Y. Wang, F. Qin, and Q. Chu, "Multi-Mode ResonatorFed Dual Polarized Antenna Array with Enhanced Bandwidth and Selectivity," IEEE Trans. Antenna Propag., vol. 63, pp. 5492-5499, 2015.

[4] C. Lin, and S. Chung, "A Filtering Microstrip Antenna Array," IEEE Trans. Microw. Theory Tech., vol. 59, pp. 2856-2863, 2011.

[5] C. Lin, and S. Chung, "A Compact Filtering Microstrip Antenna With Quasi-Elliptic Broadside Antenna Gain Response," IEEE Antennas Wireless Propag. Lett., vol. 10, pp. 381-384, 2011.

[6] C. Chuang, and S. Chung, "Synthesis and Design of a New Printed Filtering Antenna', IEEE Trans. Antenna Propag., vol. 59, pp. 1036$1042,2011$.

[7] Y. Yusuf, and X. Gong, "Compact Low-Loss Integration of High-Q 3-D Filters With Highly Efficient Antennas," IEEE Trans. Microw. Theory Tech., vol. 59, pp.857-865, 2011.

[8] A. Abbaspour-Tamijani, J. Rizk, and G. Rebeiz, "Integration of filters and microstrip antennas,” Proc. IEEE Antennas Propag. Soc. Int. Symp., pp. 874-877, 2002.

[9] J. S. Hong and M. J. Lancaster, Microwave Filger for RF/Microwave Application. Hoboken, NJ, USA: Wiley, 2001.

[10] Y. Qian, W. Deal, N. Kaneda, and T. Itoh, "Microstrip-fed quasi-Yagi antenna with broad band characteristics," Electro. Lett., vol. 34, pp. $2194-2196,1998$.

[11] G. Zheng, A. Kishk, A. Glisson, and A. Yakovlev, "Simplified feed for modified printed Yagi antenna," Electro. Lett., vol. 40, pp. 464-466, 2004. 